Artigo Original

\title{
Variabilidade de parâmetros eletromiográficos e cinemáticos em diferentes condições de marcha em idosos
}

\author{
Camilla Zamfolini Hallal \\ Nise Ribeiro Marques \\ Alex Castro \\ Deborah Hebling Spinoso \\ Denise Martineli Rossi \\ Marcelo Tavella Navega \\ José Adolfo Menezes Garcia Silva \\ Mauro Gonçalves \\ ${ }^{1}$ Instituto de Biociências, UNESP - Univ Estadual Paulista, Campus de Rio Claro, Departamento \\ de Educação Física, Laboratório de Biomecânica, Rio Claro, SP, Brasil

\begin{abstract}
Resumo: O objetivo deste estudo foi investigar a influência do medo de cair e da dupla tarefa sobre a variabilidade de parâmetros eletromiográficos e cinemáticos da marcha de idosas. Dezessete universitárias $(21,47 \pm 2,06$ anos) e dezoito idosas (65,33 $\pm 3,14$ anos), fisicamente ativas, realizaram teste de marcha em três condições: velocidade de preferência; medo de cair; e dupla tarefa. A atividade eletromiográfica dos músculos do membro inferior dominante e o comprimento e tempo de passada foram registrados. Utilizouse $o$ teste ANOVA Two-Way $(p<0,05)$. A variabilidade eletromiográfica e cinemática foram maior para 0 grupo de idosas na comparação entre grupos. Para a comparação entre as condições de marcha analisadas, houve diferença significativa apenas para variabilidade eletromiográfica. Assim, o aumento da variabilidade dos parâmetros EMG e cinemáticos do grupo de idosos em relação ao grupo de jovens sugere que o fator envelhecimento contribui para uma maior dificuldade no controle da marcha, o que possivelmente predispõe estes indivíduos à ocorrência de quedas.
\end{abstract}

Palavras-chave: Biomecânica. Eletromiografia. Marcha.

\section{Electromyographic and kinematic parameters variability in different conditions of motion in the elderly}

Abstract: The main goal of this study was to investigate the influence of fear of fall and dual task on electromyographic and kinematic variability parameters on the gait of older females. Seventeen college students $(21,47 \pm 2,06$ years old) and eighteen older female adults, both groups were physically fit and performed the gait test on three different conditions: walking at self-select speed, fear of fall and dual task. Electromyographic activity was measured on muscles of dominant leg and stride time was recorded. ANOVA two-way $(p<0.05)$ was used. Electromyographic and kinematic gait variability were higher in older adult groups. However, for the comparison between gait conditions was only found significant difference for electromyographic variability. In line with this, the higher EMG and kinematic variability in older adults suggest that aging contributes for a higher motor challenge while walking, which may be predispose these individuals a higher risk of fall.

Keywords: Biomechanics. Electromyography. Gait.

\section{Introdução}

O processo do envelhecimento é um fenômeno multifatorial que leva à redução da eficácia de um conjunto de processos fisiológicos (REBELATTO et al., 2006). Tais reduções podem levar a uma perda de independência funcional, interferindo diretamente na qualidade de vida desses idosos (REES, MURPHY e WATSFORD, 2008). A associação dessas alterações com doenças crônico degenerativas, muito prevalente nos idosos, torna esta população vulnerável a deteriorização físico-funcional (BASSEY, 1997; FARIA et al., 2003).

As alterações fisiológicas inerentes ao envelhecimento como a falta de equilíbrio, alterações na marcha, diminuição da força muscular, baixa acuidade visual, alteração da propriocepção e da cognição estão relacionadas à alta prevalência de quedas na população idosa (COLLEDGE, 1997; MENEZES; BACHION, 2008).

As causas das quedas são multifatoriais, porém mais de $50 \%$ dos casos ocorrem durante a 
marcha (MENZ; LORD; FITZPATRICK, 2003; TALBOT et al., 2005; BAIRD;RICHARD, 2009; HOLLMAN; YOUDAS; LANZINO, 2009). Deste modo, desafios cotidianos durante a marcha, podem comprometer seriamente a capacidade de controlar o equilíbrio dinâmico (PIJNAPPELS; BOBBERT; VAN DIEEN, 2001; HAHN; LEE; CHOU, 2005; VAN DIEEN; PIJNAPPELS, 2008).

Alguns autores sugerem que as modificações nos padrões de marcha em idosos devem-se principalmente ao medo de cair (REELICK et al., 2009). O medo de cair refere-se à falta de autoconfiança em realizar atividades cotidianas sem cair e ocorre com maior frequência em idosos caidores, porém, pode ser observado em idosos sem histórico de quedas (TINETTI; POWELL, 1993; SHEFFER et al., 2008; REELICK et al., 2009). Esta condição de medo pode comprometer a qualidade de vida das pessoas idosas e aumentar o risco de quedas, pois limita a mobilidade, reduz o nível de atividade independente e causa alterações na marcha (ARFKEN et al., 1994; FLETCHER; HIRDES, 2004; SHEFFER et al., 2008; BOYD; STEVENS, 2009).

O controle do equilíbrio e da marcha é visto, tradicionalmente, como um processo automático que requer baixos níveis de processamento cognitivo (TSANG; LEE; FU, 2008). Porém, estudos recentes mostram que a marcha é uma tarefa motora extremamente complexa, composta por uma sequência de movimentos cíclicos dos membros inferiores que proporcionam 0 deslocamento do corpo; e que para sua execução de maneira adequada, funções cognitivas complexas são exigidas (KERR; CONDON; McDONALD, 1985; LAJOIE et al 1993; WOOLLACOT; SHUMWAY-COOK, 2002; KIRKWOOD et al., 2007; ABREU; CALDAS, 2008; TSANG; LEE; FU, 2008; ABBUD; LI; DEMONT, 2009). Beauchet et al. (2005a) e Schultz, Lloyd e Lee (2010) apontam que tarefas cognitivas executadas simultaneamente a tarefas motoras podem alterar o controle postural de idosos e aumentar o risco de quedas nesta população.

A identificação de parâmetros biomecânicos indicadores de alterações na marcha de idosos são imprescindíveis para otimizar estratégias de prevenção à quedas nesta população. As medidas de variabilidade dos parâmetros de marcha têm sido relatadas como um excelente preditor de quedas e declínio da mobilidade (BERLIN et al., 2005; BRACH et al., 2007; ZIJLSTRA; AMINIAN, 2007; NAJAFI et al., 2009; CALLISAYA et al., 2010). O aumento da variabilidade do padrão de marcha pode ser ocasionado por diversas situações, entre elas a execução simultânea de tarefas cognitivas durante a marcha (BEAUCHET et al., 2005a).

A análise biomecânica da marcha baseada em parâmetros cinemáticos e eletromiográficos, tem se mostrado eficiente na identificação de alterações nos padrões de movimento e no comportamento neuromuscular de idosos. Deste modo, o objetivo deste estudo foi analisar a influência do medo de cair e da dupla tarefa sobre a variabilidade de variáveis cinemáticas e eletromiográficas da marcha de idosos. Com subsídio das fundamentações teóricas descritas, hipotetizamos que diferentes desafios impostos durante a marcha alteram a variabilidade de indicadores biomecânicos associados ao aumento do risco de quedas.

\section{Métodos}

\section{Participantes}

Participaram do estudo 35 voluntários. Os participantes foram divididos de acordo com a faixa etária em 2 grupos: grupo de jovens e grupo de idosos. O grupo de jovens foi composto por 17 participantes do gênero feminino, fisicamente ativas, estudantes universitárias. O grupo de idosos foi composto por 18 participantes do gênero feminino, fisicamente ativas, baixo risco de quedas e participantes de um grupo comunitário de atividade física para idosos. As voluntárias foram classificadas quanto ao nível de atividade física segundo American College of Sports Medicine, que classifica como indivíduos ativos aqueles que realizam atividade física moderada por um tempo mínimo de 30 minutos, cinco vezes por semana; ou atividade física intensa por 20 minutos, duas vezes por semana (HASKELL et al., 2007).

As participantes do grupo de idosos foram classificados quanto ao risco de quedas por meio da Escala de Equilíbrio de Berg. Esta escala é constituída por 14 tarefas que envolvem o equilíbrio estático e dinâmico em tarefas funcionais e a pontuação final obtida pela escala prediz o risco de quedas dos idosos avaliados (BEAUCHET et al., 2005b). A Tabela 1 mostra as características das voluntárias. 
Tabela 1. Características das voluntárias

\begin{tabular}{cccl}
\hline & Jovens & Idosas & \multicolumn{1}{c}{$p$} \\
\hline Idade (anos) & $21,47( \pm 2,06)$ & $65,33( \pm 3,14)$ & $<0,001^{*}$ \\
Massa $(\mathrm{Kg})$ & $60,68( \pm 5,93)$ & $64,00( \pm 1,55)$ & 0,23 \\
Estatura $(\mathrm{m})$ & $1,63( \pm 0,05)$ & $1,55( \pm 0,05)$ & $<0,001^{*}$ \\
Velocidade Preferência $(\mathrm{m} / \mathrm{s})$ & $0,95( \pm 0,84)$ & $0,70( \pm 1,92)$ & $<0,001^{*}$ \\
EEFB & - & $52( \pm 1,5)$ & - \\
\hline
\end{tabular}

${ }^{*}$ Diferenças significativas entre jovens e idosas $(p<0,05)$, EEFB (Escala de Equilíbrio Funcional de Berg).

Os critérios de exclusão adotados foram a presença de dor, fratura, ou lesão grave em músculos, tendões e/ou ligamentos nos 6 meses pregressos ao estudo, bem como histórico de alterações neurológicas, cardiovasculares ou respiratórias. Os voluntários que relataram tontura ou mal estar durante a realização dos testes também foram excluídos da amostra. O presente estudo foi aprovado em comitê de ética local e todos os participantes assinaram o termo de consentimento livre e esclarecido.

\section{Instrumentação}

Para a coleta dos dados eletromiográficos foi utilizado o módulo de aquisição de sinais biológicos por telemetria Myoresearch $\left(\right.$ Noraxon $^{\circledR}$ ) de 16 canais e software Myoresearch (Noraxon ${ }^{\circledR}$ ). As coletas dos dados cinemáticos foram feitas no plano sagital por meio de uma câmera digital (Panasonic $^{\circledR}$ NVGS 320) com frequência de

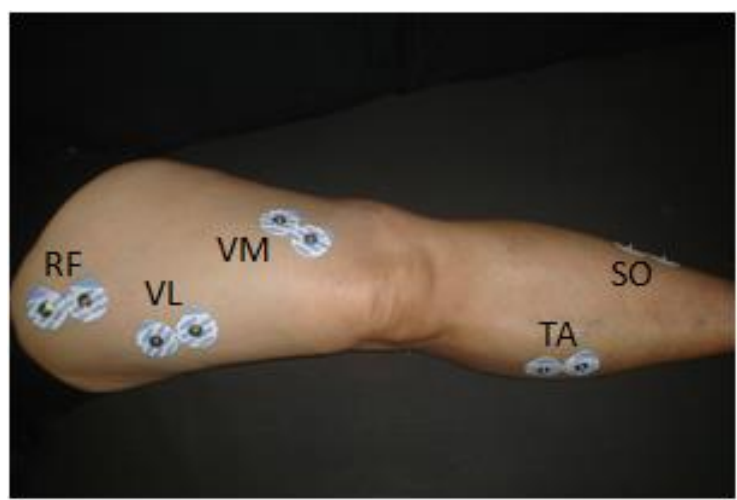

amostragem de 100 quadros/segundo e software de análise de imagem (Vicon ${ }^{\circledR} 9.0$ - Peak Motus). Os testes de marcha foram realizados em uma esteira ergométrica Millennium Super ATL $\left(\right.$ INBRAMED $\left.^{\circledR}\right)$.

\section{Procedimentos}

Os sinais eletromiográficos foram coletados durante todo o teste de marcha. Eletrodos de superfície $\mathrm{Ag} / \mathrm{AgCl}$ (Meditrace ${ }^{\circledR}$ ) foram dispostos em configuração bipolar no membro inferior dominante sobre os músculos reto femoral (RF), vasto lateral $(\mathrm{VL})$, vasto medial $(\mathrm{VM})$, bíceps femoral $(\mathrm{BF})$, tibial anterior $(\mathrm{TA})$, gastrocnêmio lateral (GL) e sóleo (SO), conforme proposto por SENIAM, como ilustra a Figura 1 (HOFFMAN E PAYNE, 1995, HERMENS et al., 2000). Os dados cinemáticos foram coletados no plano sagital com marcadores reflexivos posicionados no maléolo lateral direito e entre o primeiro e segundo metatarsos direito e esquerdo.

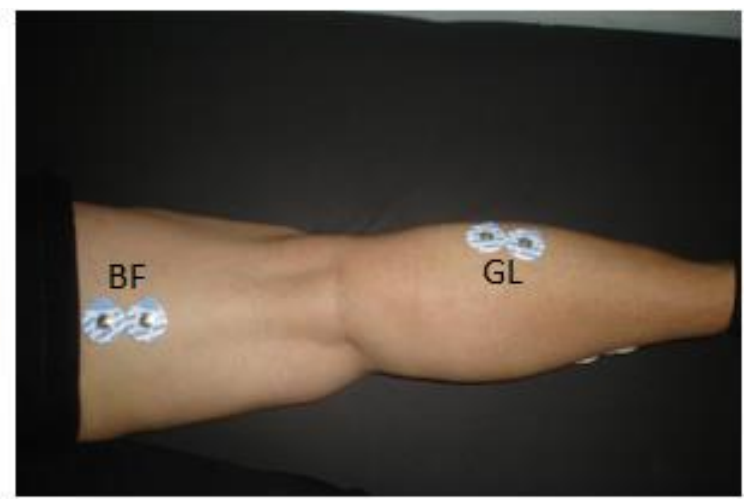

Figura 1. Posicionamento dos eletrodos nos músculos reto femoral (RF), vasto lateral (VL), vasto medial (VM), tibial anterior (TA), sóleo (SO), bíceps femoral (BF) e gastrocnêmio lateral (GL).

Antes do início do teste de marcha foi realizada a determinação da velocidade de preferência de marcha na esteira, segundo a adaptação do protocolo de Dingwell e Marin
(2006). Nesta velocidade, as voluntárias foram familiarizadas por dez minutos. Após a familiarização foi iniciado o teste de marcha em três condições distintas: marcha normal (I), 
marcha com medo de cair (II) e marcha com tarefa dupla (III). Durante a marcha I, as voluntárias foram solicitadas a andar na esteira conforme realizam suas atividades de vida diária, em velocidade de preferência por 3 minutos consecutivos. Imediatamente após a realização da marcha I, as voluntárias foram orientadas a prestar mais atenção, pois nos 3 minutos subsequentes apareceriam na esteira obstáculos que poderiam provocar tropeços. Esta condição foi caracterizada como marcha II, pois as voluntárias realizaram uma marcha em condição apreensiva, porém nenhum obstáculo apareceu durante o teste. A marcha III foi realizada logo após a marcha II. Nesta condição, as voluntárias foram instruídas a caminhar sobre a esteira em velocidade de preferência e ao mesmo tempo prestar atenção em um sistema aleatório de lâmpadas coloridas, similar a um semáforo. À medida que as lâmpadas eram acesas, as voluntárias deveriam falar as respectivas cores.

\section{Análise dos dados}

Para a análise dos dados eletromiográficos e cinemáticos foram usados os 10 ciclos consecutivos de marcha iniciais da condição normal, com medo de cair e com dupla tarefa. $O$ início e o final dos ciclos foram determinados pelo momento de maior distância entre o ponto reflexivo do maléolo direito e metatarso esquerdo, no momento do toque do calcâneo direito, de acordo com protocolo adaptado de Kang e Dingwell (2008), ilustrado na Figura 2.

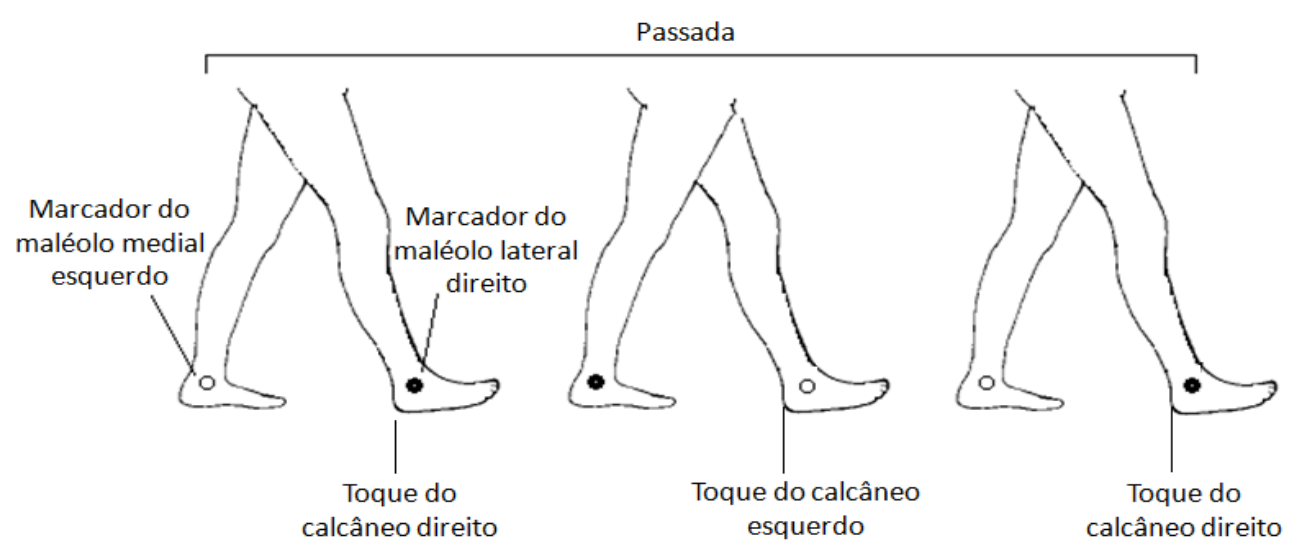

Figura 2. Esquema ilustrativo demonstrando o início e o fim de marcha.

Os sinais eletromiográficos foram coletados com frequência de amostragem de $1000 \mathrm{~Hz}$ e processados por meio de rotinas desenvolvidas em ambiente MatLab $7.0^{\circledR}$. Foi utilizado filtro passa alta de $20 \mathrm{~Hz}$, passa baixa de $500 \mathrm{~Hz}$, filtro notch de $60 \mathrm{~Hz}$ e filtro de $6 \mathrm{~Hz}$ para a criação do envelope linear. Os dados retificados foram interpolados em 1001 pontos sobre os 10 ciclos de marcha em cada condição. O cálculo da média dos valores obtidos durante os 10 ciclos de marcha na condição normal foi utilizado para a normalização dos valores de envelope linear para cada sujeito e músculo analisado.

As variáveis cinemáticas analisadas foram o comprimento de passada e o tempo de passada. Os dados foram processados por meio do software de análise de imagem Peak Motus (Vicon $^{\circledR}$ 9.0). Os valores de comprimento de passada foram normalizados pelo tamanho do membro inferior direito de cada voluntária.
A análise da variabilidade foi realizada pelo cálculo da média dos desvios padrão para cada variável analisada (DINGWELL; MARTIN, 2006; KANG; DINGWELL, 2008; KANG; DINGWELL, 2009).

A análise estatística foi realizada por meio do software PASW statistics $18.0^{\circledR}$. Para a análise dos dados de tempo de passada, comprimento de passada e ativação muscular, foi utilizado o teste ANOVA TwoWay e o teste Post-Hoc Bonferroni para as comparações entre os grupos e entre as condições de marcha.

\section{Resultados}

\section{Variabilidade Eletromiográfica}

A tabela 2 mostra os valores referentes à variabilidade de ativação dos músculos $R F, V M$, VL, BF, TA, GL e SO na comparação entre os grupos, jovens e idosos, e entre as condições de marcha I, II e III. 
Em relação à análise da comparação entre os grupos, houve diferença significativa para todos os músculos analisados, sendo que os valores de $F$ e $p$ foram: $R F(p<0,001 ; F=10400,420), V M$ $(p<0,001 ; \quad F=5325,743), \quad V L \quad(p<0,001$; $F=5319,799), \quad B F \quad(p<0,001 ; \quad F=3071,726), \quad T A$ $(p<0,001 ; F=510,635), G L(p<0,001 ; F=279,073)$ e SO ( $p<0,001 ; F=316,501)$.

A análise da interação entre as condições mostrou que houve diferença significativa para os músculos $R F(p<0,001 ; F=10,630)$, VM $(p<0,001$; $F=9,373), B F(p<0,001 ; F=7,930)$ e $G L(p=0,002$; $F=7,073)$.

Em relação à análise da interação entre grupos $X$ condições de marcha, não foi encontrada diferença para a variabilidade eletromiográfica dos músculos analisados.

\section{Variabilidade Cinemática}

A tabela 2 mostra a comparação da variabilidade do tempo e do comprimento de passada entre os grupos de jovens e idosas nas diferentes condições de marcha.

Houve diferença entre os grupos para a variabilidade do tempo de passada, sendo este menor para o grupo de jovens $(p=0,004$, $\mathrm{F}=8,451)$. Não foram encontradas diferenças de variabilidade de tempo de passada entre as condições de marcha analisadas $(p=0,896)$.

Tabela 2. Variabilidade eletromiográfica e cinemática de jovens e idosas em diferentes condições de marcha

\begin{tabular}{|c|c|c|c|c|c|c|c|c|c|}
\hline \multirow{2}{*}{ Variabilidade } & \multicolumn{3}{|c|}{ Idosas } & \multicolumn{3}{|c|}{ Jovens } & \multicolumn{3}{|c|}{ Efeitos Principais (P-Valores) } \\
\hline & $\mathrm{Cl}$ & Cll & CIII & $\mathrm{Cl}$ & CII & CIII & Grupo & Condição & $\begin{array}{c}\text { Grupo X } \\
\text { Condição }\end{array}$ \\
\hline \multicolumn{10}{|l|}{ EMG Músculos } \\
\hline RF & $28,4 \pm 0,8^{*}$ & $28,6 \pm 1,6^{\star}$ & $30,0 \pm 1,1$ & $2,8 \pm 0,2^{*}$ & $3,3 \pm 0,3^{*}$ & $3,9 \pm 0,2$ & $<0,001$ & $<0,001$ & 0,341 \\
\hline VM & $29,9 \pm 1,1^{\star \#}$ & $32,0 \pm 1,7$ & $31,9 \pm 1,4$ & $5,5 \pm 0,7^{\star \#}$ & $6,5 \pm 1,1$ & $6,8 \pm 0,6$ & $<0,001$ & $<0,001$ & 0,415 \\
\hline VL & $34,0 \pm 1,0^{\star \#}$ & $36,0 \pm 2,7$ & $35,9 \pm 1,3$ & $7,0 \pm 0,3^{\star \#}$ & $8,1 \pm 0,6$ & $8,2 \pm 0,3$ & $<0,001$ & 0,002 & 0,589 \\
\hline $\mathrm{BF}$ & $32,6 \pm 1,8^{\star \#}$ & $34,9 \pm 1,7$ & $34,4 \pm 1,2$ & $13,3 \pm 0,9^{\star \#}$ & $14,4 \pm 0,7$ & $13,9 \pm 0,7$ & $<0,001$ & 0,001 & 0,260 \\
\hline TA & $35,1 \pm 2,9$ & $37,2 \pm 3,0$ & $36,2 \pm 1,7$ & $20,3 \pm 1,8$ & $20,7 \pm 1,4$ & $21,7 \pm 2,6$ & $<0,001$ & $<0,206$ & 0,432 \\
\hline GL & $34,3 \pm 2,6^{\star \#}$ & $36,3 \pm 2,8$ & $35,3 \pm 1,8$ & $24,4 \pm 1,1^{\text {*\# }}$ & $26,8 \pm 1,2$ & $27,6 \pm 0,8$ & $<0,001$ & 0,002 & 0,224 \\
\hline so & $32,0 \pm 1,0$ & $32,9 \pm 2,3$ & $33,1 \pm 1,9$ & $23,8 \pm 0,8$ & $24,3 \pm 1,3$ & $25,3 \pm 0,8$ & $<0,001$ & 0,09 & 0,780 \\
\hline \multicolumn{10}{|l|}{ Cinemática } \\
\hline TP & $0,04 \pm 0,02$ & $0,04 \pm 0,02$ & $0,04 \pm 0,03$ & $0,03 \pm 0,01$ & $0,03 \pm 0,01$ & $0,03 \pm 0,01$ & 0,004 & 0,896 & 0,986 \\
\hline $\mathrm{CP}$ & $5,50 \pm 1,97$ & $6,42 \pm 2,68$ & $6,58 \pm 3,07$ & $4,98 \pm 1,67$ & $4,98 \pm 1,57$ & $5,17 \pm 1,53$ & 0,007 & 0,433 & 0,590 \\
\hline
\end{tabular}

Abreviações: Reto Femoral (RF), Vasto Medial (VM), Vasto Lateral (VL), Bíceps Femoral (BF), Tibial Anterior (TA), Gastrocnêmio Lateral (GL), Sóleo (SO), Tempo de Passada (TP), Comprimento de Passada (CP). *Diferença significativa em relação à Condição III, "Diferença significativa em relação à Condição II.

A variabilidade do comprimento de passada foi menor para $o$ grupo de jovens $(p=0,007$, $F=7,515)$. No entanto, não houve diferença entre as condições de marcha analisadas $(p=0,433)$.

$\mathrm{Na}$ análise da interação entre grupos $X$ condições de marcha não foi encontrada diferença para a variabilidade do tempo e comprimento de passada $(p=0,986$ e $p=0,590$, respectivamente).

\section{Discussão}

Instabilidades posturais durante a marcha são comumente observadas na população idosa e estão associadas a uma combinação de fatores inerentes ao envelhecimento, estando, portanto, sobre foco de diversos estudos (TINETTI; SPEECHLEY, 1989; LORD; DAYHEW, 2001; PERRACINI; RAMOS, 2002; HORTOBÁGYI et al., 2009; ROCHAT et al., 2010). A adoção de estratégias adaptativas no andar do idoso surge mediante a necessidade de compensar os déficits neuromusculares, como, por exemplo, a diminuição da velocidade, menor comprimento de passo, redução do tempo da fase de balanço e diminuição dos movimentos articulares do quadril, joelho e tornozelo (HAGEMAN et al., 1986; JUFGE; DAVIS; OUNPUU; 1996; $\underline{\mathrm{CHEN}}$ et al., 1991; DeVITA; HORTOBÁGYI, 2000; HAHN; CHOU, 2004; HAHN; LEE; CHOU, 2005; HORTOBÁGYI et al., 2009). 
Haja vista que a marcha não pode ser considerada um ato reflexo e sim um processo que requer associação de respostas cognitivas para gerar uma execução adequada, objetivamos com o presente estudo analisar a influência do medo de cair e da dupla tarefa sobre a variabilidade de variáveis cinemáticas e eletromiográficas da marcha de idosos para compreender os efeitos de sobrecargas relacionadas à tarefa durante 0 processo de deambulação (LUNDIN-OLSSON; NYBERG; GUSTAFSON, 1997; BEAUCHET et al., 2003; HOLLMAN; SALAMON; PRIEST, 2004).

As medidas de variabilidade dos parâmetros de marcha têm despertado grande interesse nos pesquisadores por tratar-se de um excelente preditor de quedas e declínio da mobilidade (BRACH et al., 2008; BRACH et al., 2007; CALLISAYA et al., 2009; NAJAFI et al., 2009). Altos valores de variabilidade no andar referem-se à flutuação nos valores dos parâmetros de marcha de uma passada para outra e é considerado um indicativo de instabilidade, pois reflete distúrbios no controle motor, como resultado de déficits no sistema nervoso central e periférico (HAUSDORFF, 2007; BRACH et al., 2008; BEAUCHET et al., 2009; CALLISAYA et al., 2009). O aumento da variabilidade do padrão de marcha pode ser ocasionado por diversas situações, entre elas a execução simultânea de tarefas cognitivas durante a marcha (BEAUCHET et al., 2005a).

Nossos resultados mostram que houve diferença significativa entre idosos e jovens para a variabilidade do comprimento e do tempo de passada e da variabilidade de ativação muscular. Os mecanismos que geram ativações musculares para a execução da marcha normalmente envolvem a geração de padrões centrais e feedback sensorial. Com o avanço da idade, estas funções podem deteriorar-se e serem pouco eficazes nas respostas à perturbações e restauração da marcha funcional (NAYFEH; BALACHANDRAN, 1995; IVANENKO et al., 2006; KANG; DINGWELLS, 2009). Deste modo, os altos valores de variabilidade eletromiográfica e cinemática durante $\mathrm{o}$ andar encontrados neste estudo, podem ser considerados indicativos de instabilidade, pois refletem distúrbios no controle motor como resultado de déficits no sistema nervoso central e periférico decorrentes do envelhecimento (KANG; DINGWELLS, 2009).
A estabilidade cinemática da marcha requer a geração de padrão motor apropriado e respostas corretivas efetivas frente à perturbações (DINGWELL; MARTIN, 2006). Assim, uma vez que os músculos são responsáveis por gerar as forças que comandam a execução da marcha, a cinemática presumidamente reflete os padrões de ativação muscular (DINGWELL; MARTIN, 2006). Entretanto, os parâmetros cinemáticos são muito menos variáveis que os eletromiográficos e os padrões de cinemática parecem mais rigidamente controlados do que a ativação muscular. Tal fato pode estar relacionado principalmente às propriedades de inércia e amortecimento dos segmentos corporais o que explica a menor variabilidade encontrada nos dados cinemáticos em relação aos dados eletromiográficos (DINGWELL; MARTIN, 2006).

No entanto, em estudos realizados por Reelick et al. (2009) com idosos com medo de cair e por Montero-Odasso et al. (2009) com idosos em situação de dupla tarefa foram encontrados aumento na variabilidade de diversos parâmetros cinemáticos como comprimento de passada, tempo de duplo apoio, tempo de passada e tempo de passo.

Apesar do aumento da variabilidade em variáveis cinemáticas ser considerado um indicativo de que a divisão da atenção em outras tarefas que não a própria marcha, requer modulações mais complexas do sistema neuromotor do que a marcha isolada de outros estímulos, nos resultados apresentados não foram encontradas estas diferenças (BEAUCHET et al., 2005b; SCHULTZ; LLOYD; LEE, 2010). Tais achados podem estar relacionados principalmente ao fato de que, no presente estudo, as condições de marcha com dupla tarefa caracterizaram-se pela indução de estímulos dados às voluntárias em ambiente laboratorial, além disto, todas as participantes eram saudáveis, ativas e com baixo risco de quedas.

\section{Limitações do Estudo}

A velocidade de preferência foi significativamente diferente entre jovens e idosos. Deste modo, o desafio mecânico imposto às articulações dos participantes pode ter sido diferente. No entanto, a velocidade de preferência foi escolhida por tratar-se da velocidade aproximada em que cada voluntária caminha em suas atividades cotidianas, sendo, portanto, considerada ideal para a avaliação das mudanças 
nos parâmetros de marcha advindas do medo de cair.

\section{Conclusão}

Os desafios impostos durante a marcha, tal como o medo de cair e a marcha com identificação de estímulo luminoso, aumentaram a variabilidade EMG em relação à marcha normal, demonstrando a importância da função muscular para a manutenção da estabilidade das articulações do quadril e joelho durante a marcha.

O aumento da variabilidade dos parâmetros EMG e cinemáticos do grupo de idosos em relação ao grupo de jovens sugere que o fator envelhecimento contribui para uma maior dificuldade no controle da marcha, o que possivelmente predispõe estes indivíduos à ocorrência de quedas.

\section{Referências}

ABBUD, G. A. C.; LI, K. Z. H.; DEMONT, R. G. Attentional requirements of walking according to the gait phase and onset of auditory stimuli. Gait \& Posture, Amsterdam, v. 30, p. 227-232, 2009.

ABREU, S. S. E. ; CALDAS, C. P.; Velocidade de marcha, equilíbrio e idade: um estudo correlacional entre idosas praticantes e não praticantes de um programa de exercícios terapêuticos. Revista Brasileira de Fisioterapia, São Carlos, v. 12, n. 4, p. 324-330, 2008.

ARFKEN, C. L.; LACH, H. W.; BIRGE, S. J.; MILLER, J. P. The prevalence and correlates of fear of falling in elderly persons living in the community. American Journal of Public Health, New York, v. 84, p. 565-570, 1994.

BAIRD, J. L; RICHARD, E. A. Young and older adults use different strategies to perform a standing turning task. Clinical Biomechanics, Bristol, v. 24, p. 826-832, 2009.

BASSEY, E. J. Physical capabilities, exercise and aging. Reviews in Clinical Gerontology, London, v. 7, p. 289-297, 1997.

BEAUCHET, O.; ALLALI, G.; ANNWEILER, C.; BRIDENBAUGH, S.; ASSAL, F.; KRESSIG, R. W.; HERRMANN, F. R. Gait variability among healthy adults: low and high stride-to-stride variability are both a reflection of gait stability. Gerontology, Basel, v. 55, p. 702-706, 2009.

BEAUCHET, O.; DUBOST, V.; AMINIAN, K.; GONTHIER, R.; KRESSIG, R. W. Dual-task- related gait changes in the elderly: does the type of cognitive task matter? Journal Motor

Behavior, Washington, v. 37, p. 259-264, 2005a.

BEAUCHET, O.; DUBOST, V.; HERRMANN, F. ; RABILLOUD, M.; CONTHIER, R.; KRESSIG, R. W. Relationship between dual-task related gait changes and intrinsic risk factors for falls among transiotional frail older adults. Aging Clinical and Experimental Research, Milano, v. 17, p. 270275, 2005b.

BEAUCHET, O.; KRESSIG, R. W.; NAJAFI, B.; AMINIAN, K.; DUBOST, V.; MOUREY, F. Agerelated decline of gait control under a dual-task condition. J Journal of the American Geriatrics Society, New York, v. 51, p. 1187-1188, 2003. .

BERLIN, J. E.; Van SWCARINGER, J. M.; NEWMAN, A. B.; STUDENSKI, S. A. Too much or too little step width variability is associated with a fall history in older person who walk at or near normal gait speed. Journal Neuroengineering Rehabilitation, London, v. 2, p. 21, 2005.

BLAKE, A.; MORGAN, M.; DALLOSSO, H.; EBRAHIM, S.; ARIE, T.; FENTEM, P.; BASSEY, $E$. Falls by elderly people at home: prevalence and associated factors. Age and Ageing, London, v.17, p. 365-372, 1988.

BOYD, R.; STEVENS, J. A. Falls and fear of falling: burden, beliefs and behaviours. Age and Ageing, London, v. 38, p. 423-428, 2009.

BRACH, J. S.; STUDENSKI, S.; PERERA, S.; VANSWCARINGER, J. M.; NEWMAN, A.B. Stance time and step width variability have unique contribuition impairments in older person. Gait \& Posture, Amsterdam, v. 27, p. 431-439, 2008.

BRACH, J. S.; STUDENSKI, S. A.; VAN SWCARINGER, J. M.; NEWMAN, A. B. Gait variability and the risk of incident mobility disability in community-dwelling older adults. Journal of Gerontology: Biology Sciences, Washington, v. 62, p. 983-988, 2007.

CALLISAYA, M. L.; BLIZZARD, L.; SCHMIDT, M. D.; MCGINLEY, J. L.; SRIKANTH, V. K. Ageing and gait variability-a population-based study of older people. Age and Ageing, London, v. 39, p. 191-197, 2010.

CHAMBERS, A. J., CHAN, R. Slip-related muscle activation patterns in the stance leg during walking. Gait \& Posture, Amsterdam, v. 25, p. 565-572, 2007.

CHEN, H. C.; ASTON-MILLER, J. A.; ALEXANDER, N. B.; SCHULTZ, A. B. Stepping 
over obstacles: gait patterns of healthy yong and old adults. Journal of Gerontology, Washington, v. 46, p. 196- 203, 1991.

\section{COLLEDGE, N. Falls. Review In Clinical} Gerontology, London, v. 7, p. 309-315, 1997.

DeVITA, P.; HORTOBÁGYI, T. Age causes a redistribution of joint torques and powers : during gait. Journal of Applied Physiology, Washington, v. 88, p. 1804-1811, 2000.

DINGWELL, J. B.; MARTIN, L. C. Kinematic variability and local dynamic stability of upper body motions when walking at different speeds. Journal of Biomechanics, Elmsford, v. 39, p. 444-452, 2006.

FARIA, J. C.; MACHALA, C. C.; DIAS, R. C.; DIAS, J. M. D. Importância do treinamento de força na reabilitação da função muscular, equilíbrio e mobilidade de idosos. Acta Fisiátrica, São Paulo, v. 10, p. 133-137, 2003.

FLETCHER, P. C.; HIRDES, J. P. Restriction in activity associated with fear of falling among community-based seniors using home care services. Age and Ageing, London, v. 33, p. 273279, 2004.

HAGEMAN, P. A.; BLANKE, D. J. Comparison of gait of young women and elderly women.

Physical Therapy, New York, v. 66, p. 13821387, 1986.

HAHN, M. E.; LEE, H. J.; CHOU, L. S. Increased muscular challenge in older adults during obstructes gait. Gait \& Posture, Amsterdam, v. 22, p. 356-361, 2005.

HAHN, M. E.; CHOU, L. S. Age- related reduction in sagittal center of mass motion during obstacle crossing. Journal of Biomechanics, Elmsford, v. 37, p. 837-844, 2004.

HASKELL, W. L.; LEE, I. M.; PATE, R. R.; POWELL, K. E.; BLAIR, S. N.; FRANKLIN, B. A.; MACERA, C. A.; HEATH, G. W.; THOMPSON, P. D.; BAUMAN, A. Physical Activity and Public Health Updated Recommendation for Adults From the American College of Sports Medicine and the American Heart Association. Medicine Sciense Sports Exercise, Medison, v. 39, p. 1423-34, 2007.

HAUSDORFF, J. M. Gait dynamics, fractals and falls: finding meaning in the stride-tostride fluctuations of human walking. Human Movement Science, Amsterdam, v. 26, p. 555-589, 2007.
HERMENS, J. H.; FRERIKS, B.; DISSELHORSTKLUG, C.; RAU, G. Development of recommendations for SEMG sensors and sensor placement procedures. Journal Electromyography Kinesiology, New York, v. 10, p. 361-374, 2000.

HOLLMAN, J. H; YOUDAS, J, W; LANZINO, D. J. Gender differences in dual task gait performance in older adults. American Journal of Men's Helth, New Orleans, v. 22, 2009.

HOLLMAN, J. H.; SALAMON, K. B.; PRIEST, A. W. Age-related differences in stride-to-stride variability during dual task walking: a pilot study. Journal Geriatric Physical Therapy, La Crosse, v. 27, n. 3, p. 83-87, 2004.

HORTOBÁGYI, T.; SOLNIK, S., GRUBER, A.; RIDER, P.; STEINWEG, K.; HELSETH, J.; DEVITA, $P$. Interaction between age and gait velocity in the amplitude and timing of antagonist muscle coactivation. Gait \& Posture, Amsterdam, v. 29, p. 558-564, 2009.

IVANENKO, Y.P.; POPPELE, R.E.; LACQUANITI, F. Spinal cord maps of spatiotem- poral alphamotoneuron activation in humans walking at different speeds. Journal of Neurophysiology, Bethesda, v. 95, n. 2, p. 602-618, 2006.

JUFGE, J. O.; DAVIS, R. B.; OUNPUU, S. Step length redutions in advanced age: the role of ankle and hip kinetics. Journal of Gerontology, Washington, v. 51, p. 303- 312, 1996.

KANG, H. G.; DINGWELL, J. B. Dynamics and stability of muscle activations during walking in healthy young and older adults. Jounal of Biomechanics, Elmsford, v. 42, p. 2231-2237, 2009.

KANG, H. G.; DINGWELL, J. B. Separating the effects of age and walking speed on gait variability. Gait \&Posture, cidade, v. 27, p. 572577, 2008.

KERR, B.; CONDON, S. M.; McDONALD, L. A. Cognitive spatial processing and the regulation of posture. Journal of Experimental Psychology: Human Perception and Performance, Washington, v. 11, p. 617-622, 1985.

KIRKWOOD, R. N.; GOMES H. A.; SAMPAIO, R. F.; CULHAM, E.; COSTIGAN, P. Análise biomecânica das articulações do quadril e joelho durante a marcha em participantes idosos. Acta Ortopédica Brasileira, São Paulo, v. 15, n. 5, p. 267-271, 2007. 
LAJOIE, Y.; TEASDALE, N.; BARD, C.; FLEURY, M. Attentional demands for static and dynamic equilibrium. Experimental Brainz Reserch, Berlin, v. 1, p. 139-144, 1993.

LORD, S. R.; DAYHEW, J. Visual factors for falls in older people. Journal American Geriatric Society, v. 49, p. 508-515, 2001.

LUNDIN-OLSSON, L.; NYBERG, L.;

GUSTAFSON, Y. "Stops walking when talking" as a predictor of falls in elderly people. Lancet, London, v. 349, p. 617, 1997.

MENZ, H. B.; LORD, S. R.; FITZPATRICK, R. C. Acceleration patterns of the head and pelvis when walking on level and irregular surfaces. Gait \& Posture, Amsterdam, v.18, p. 35-46, 2003.

MENEZES, R. L.; BACHION, M. M. Estudo da presença de fatores de riscos intrínsecos para quedas, em idosos institucionalizados. Ciência \& Saúde Coletiva, Rio de Janeiro, v.13, n.4, p.1209-1218, 2008.

MONTERO-ODASSO, M.; CASAS, A.; HANSEN, K. T.; BILSKI, P.; GUTMANIS, I.; WELLS, J. L.; BORRIE, M. J. Quantitative gait analysis under dual-task in older people with mild cognitive impairment: a reliability study. Journal of NeuroEngineering and Rehabilitation, London, v. 6 , p. 35-40, 2009.

NAJAFI, B.; HELBOSTAD, J.; MOE-NILSSEN, R.; ZIJLSTRA, W.; AMINIAN, K. Does walking strategy in older people change as a function of walking distance? Gait \& Posture, Amsterdam, v. 29, p. 261-266, 2009.

NAYFEH, A. H.; BALACHANDRAN, B. Applied Nonlinear Dynamics: Analytical, Computational, and Experimental Methods. Wiley, New York, 1995.

PERRACINI, M. R.; RAMOS, L. R. Fatores associados a quedas em uma coorte de idosos residentes na comunidade. Revista de Saúde Pública, São Paulo, v. 36, n. 6, p. 709-716, 2002.

PIJNAPPELS, M.; BOBBERT, M. F.; VAN DIEEN, J. H. Changes in walking pattern caused by the possibility of a tripping reaction. Gait \& Posture, Amsterdam, v. 14, p. 11-18, 2001.

REBELLATO, J. R.; CALVO, J. I.; OREJUELA, J. R.; PORTILLO, J. C. Influência de um programa de atividade física de longa duração sobre a força muscular manual e a flexibilidade corporal de mulheres idosas. Rev Bras Fisioter, São Carlos, v. 10, n. 1, p. 127-132, 2006.
REELICK, M. F.; VAN IERSEL, M. B.; KESSELS, R. P.; RIKKERT, M. G. The influence of fear of falling on gait and balance in older people. Age and Ageing, London, v. 38, p. 435-440, 2009.

REES, S.; MURPHY, A.; WATSFORD, M. Effects of vibration exercise on muscle performance and mobility in an older population. Journal of Aging and Physical Activity, Champaign, v. 15, n. 4, p. 367-381, 2008.

ROCHAT, S.; BÜLA, C. J.; MARTIN, E.; SEEMATTER-BAGNOUD, L.; KARMANIOLA, A.; AMINIAN, K.; PIOT-ZIEGLER, C.; SANTOSEGGIMANN, B. What is the relationship between fear of falling and gait in well-functioning older persons aged 65 to 70 years? Archives of Physical Medicine and Rehabilitation, Chicago, v. 91 , p. $879-884,2010$.

SHEFFER, A. C.; SCHUURMANS, M. J.; DIJK, N.; HOOFT, T.; ROOJI, S. E. Fear of falling: measurement strategy, prevalence, risk factors and consequences among older persons. Age and Ageing, London, v. 37, p. 19-24, 2008.

SCHULTZ, B. W.; LLOYD, J. D.; LEE, W. E. The effects of everyday concurrent tasks on overgroun minimum toe clearance and gait parameters. Gait \& Posture, Amsterdam, v. 32, n.1, p.18-22, mai. 2010.

TALBOT, L. A; MUSIOL, R. J.; WITHAM, E.K.; METTER, E. J. Falls in young, middle-aged and older community dwelling adults: perceived cause, environmental factors and injury. BCM Public Health, London, v. 5, p. 86-90, 2005.

TINETTI, M.; SPEECHLEY, M. Prevention of falls among the elderly. New England Journal of Medicine, Boston, v. 320, p.1055-1059, 1989.

TINETTI, M. E.; POWELL, L. Fear of falling and low self-efficacy: a case of dependence in elderly persons. Journal of Gerontology, Washington, v. 48, p. 35-38, 1993.

TSANG, W. W.; LEE, K. Y.; FU, A. S. Effects of concurrent cognitive task on pre-landing muscle response latency during stepping down activity in older adults with and without a history of falls. Disability and Rehabilitation, London, v. 30, p. 1116-1122, 2008.

VAN DIEEN, J. H.; PIJNAPPELS, M. Falls in older peoples. Journal of Electromyography and Kinesiology, New York, v. 18, p.169-171, 2008.

WATT, J.; FRANZ, J. R.; JACKSON, K.; DICHARRY, J.; RILEY, P. O.; D. C. KERRIGAN. A three-dimensional kinematic and kinetic 
comparison of overground and treadmill walking in healthy elderly subjects. Clinical of

Biomechanics, Bristol, v. 25, p. 444-449, 2010.

WOOLLACOT, M.; SHUMWAY-COOK, A.

Attention and the control of posture and gait: a review of an emerging area of research. Gait \& Posture, Amsterdam, v. 16, p. 1-14, 2002.

ZIJLSTRA, W.; AMINIAN, K. Mobility assessment in older people: new possibilites and challenges. European Journal of Ageing, Berlin, v. 4, p. 312, 2007.

Agradecimentos: CNPq, Fapesp e Fundunesp pelo apoio financeiro.

\section{Endereço:}

Camilla Zamfolini Hallal

LAB - Depto. de Educação Física

Avenida 24 A, 1515, Bairro: Bela Vista

Rio Claro SP Brasil

13506-900

e-mail: camillazhallal@yahoo.com.br

Recebido em: 19 de setembro de 2011.

Aceito em: 18 de fevereiro de 2013.

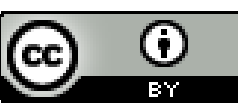

Motriz. Revista de Educação Física. UNESP, Rio Claro, SP, Brasil - elSSN: 1980-6574 - está licenciada sob Creative Commons - Atribuição 3.0 